\title{
Braiding Majorana corner modes in a second-order topological superconductor
}

\author{
Tudor E. Pahomi $\odot,{ }^{1, *}$ Manfred Sigrist, ${ }^{1}$ and Alexey A. Soluyanov ${ }^{2,3, \dagger}$ \\ ${ }^{1}$ Institute for Theoretical Physics, ETH Zurich, 8093 Zurich, Switzerland \\ ${ }^{2}$ Physik-Institut, University of Zurich, 8057 Zurich, Switzerland \\ ${ }^{3}$ Department of Physics, St. Petersburg State University, St. Petersburg 199034, Russia
}

(Received 23 April 2019; revised 1 May 2020; accepted 1 September 2020; published 15 September 2020)

\begin{abstract}
We propose the concept of a device based on a square-shaped sample of a two-dimensional second-order topological helical superconductor which hosts two zero-dimensional Majorana quasiparticles at the corners. The two zero-energy modes rely on particle-hole symmetry (PHS) and their spatial position can be shifted by rotating an in-plane magnetic field and tuning proximity-induced spin-singlet pairing. We consider an adiabatic cycle performed on the degenerate ground-state manifold and show that it realizes the braiding of the two modes whereby they accumulate a nontrivial statistical phase $\pi$ within one cycle. Alongside the PHS-ensured operator algebra, the fractional statistics confirms the Majorana nature of the zero-energy excitations. A schematic design for a possible experimental implementation of such a device is presented, which could be a step towards realizing non-Abelian braiding.
\end{abstract}

DOI: 10.1103/PhysRevResearch.2.032068

Introduction. Standard $d$-dimensional topological insulators and superconductors have a gapped bulk spectrum and exhibit conducting surface states in $(d-1)$ dimensions [1-8]. The analysis of noninteracting electrons in materials possessing fundamental symmetries-time-reversal $(\mathcal{T})$, particle-hole $(\mathcal{P})$, and/or chiral symmetry $(\mathcal{C})$-led to the initial classifications of such topological phases [9-11]. Later on, possible topological phases protected by discrete crystalline symmetries were studied [12-24] and, lastly, another class of exotic noninteracting topological phases was discovered, namely the second-order topological insulators (SOTIs) and superconductors (SOTSs) [25-31].

Unlike standard gapped topological materials (that may be termed "first order"), such systems have insulating $(d-$ 1)-dimensional surfaces, but host topologically protected boundary modes in $(d-2)$ dimensions. In particular, for $d=3$ this means the material exhibits one-dimensional (1D) states propagating along its hinges [26,27,31-37] as detected in $\mathrm{Bi}(111)$ [38]. Accordingly, SOTIs and SOTSs in $d=2$ host localized $(d=0)$ corner modes and have been discussed in both static [18,25,31-35,37,39] and Floquet-driven systems [40-42]. Second-order topological phases have been experimentally realized in artificial systems, such as mechanical metamaterials [43], microwave [44] and topolectrical circuits [45], and further implementations in materials have been proposed $[27,34,46,47]$.

\footnotetext{
"pahomit@phys.ethz.ch

${ }^{\dagger}$ Deceased.
}

Published by the American Physical Society under the terms of the Creative Commons Attribution 4.0 International license. Further distribution of this work must maintain attribution to the author(s) and the published article's title, journal citation, and DOI.
One of the motivations to seek for topological materials is their potential implementation in devices that would exploit the topology-ensured protection from decoherence. Of particular interest is topological quantum computation, considered the cornerstone of quantum technology, in which logical operations are done by means of braiding (double exchange) non-Abelian quasiparticle excitations [48-52]. Majorana (zero-energy) excitations are known to be the only non-Abelian quasiparticles that can appear in the absence of electron-electron interactions [53,54] and were initially predicted to appear at the edges of a 1D spinless superconductor [48]. Almost a decade later, it was demonstrated that this exotic topological phase could be achieved in a semiconductor nanowire with strong spin-orbit coupling placed in an external magnetic field, if superconductivity is induced by a proximity effect $[55,56]$. Following these theoretical proposals, such devices were experimentally realized by several groups [57-62] and are currently among the most promising building blocks for topological quantum computing devices. However, despite the existing proposals of braiding Majorana end modes using nanowires $[63,64]$, identifying the topological qubit remains extremely challenging due to the engineering complexity of manipulating the topological phase in the wire junctions without losing the superconducting state. This motivates the search for other approaches to realize a Majorana qubit in potentially feasible condensed matter systems [65-69].

In this Rapid Communication we propose a device based on a two-dimensional (2D) SOTS which hosts two Majorana modes localized at two corners of a square-shaped crystalline sample [see Fig. 1(a)]. We call them Majorana corner states (MCSs) in the following. Within our model, the corner localization of the MCSs in the device can be tuned using an in-plane magnetic field and proximity-induced spin-singlet superconductivity. In this setup, the braiding of the Majorana quasiparticles can be realized in a straightforward fashion 

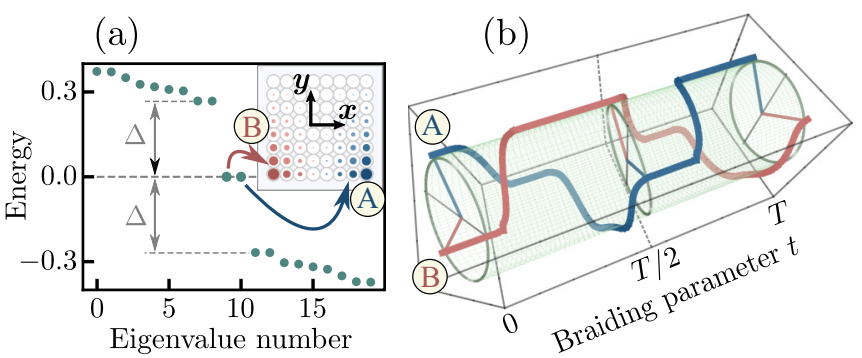

FIG. 1. (a) Energy of the lowest 20 eigenstates in a finite squareshaped sample of our second-order topological superconductor for parameters $\boldsymbol{b}=(-0.3,0)$ and $\boldsymbol{s}=(0,0.3)$; the two zero-energy modes, denoted by $\mathrm{A}$ and $\mathrm{B}$, are separated from the bulk states by an energy gap $\Delta$ and the probability densities $\left|\psi_{\alpha}(x, y)\right|^{2}$ are schematically shown in the inset $(\alpha \in\{\mathrm{A}, \mathrm{B}\})$. (b) Worldlines of A and B during the proposed cyclic adiabatic process of period $T$, computed as $\arg \max _{\varphi}\left|\psi_{\alpha}(\varphi)\right|^{2}$ for each $t \in[0, T]$, with $\varphi$ the polar angle.

[schematically illustrated in Fig. 1(b)], and we show that the associated fractional statistics of the proposed topological Majorana qubit is observed.

SOTS model. In $\boldsymbol{k}$ space, our proposed model can be formulated with a minimal four-band Bogoliubov-de Gennes (BdG) Hamiltonian

$$
\mathcal{H}(\boldsymbol{k})=\left(\begin{array}{cc}
\hat{h}_{k} & \hat{\Delta}_{k} \\
\hat{\Delta}_{\boldsymbol{k}}^{*} & -\hat{h}_{k}^{*}
\end{array}\right),
$$

written in the Nambu basis $\hat{\Psi}_{k}:=$ $\left(\begin{array}{llll}c_{\boldsymbol{k} \uparrow} & c_{\boldsymbol{k} \downarrow} & c_{-\boldsymbol{k} \uparrow}^{\dagger} & -c_{-\boldsymbol{k} \downarrow}^{\dagger}\end{array}\right)^{T}$, with $c_{\boldsymbol{k} s}^{(\dagger)}$ being the annihilation (creation) operator for an electron with a 2D momentum $\boldsymbol{k}$ and spin $s \in\{\uparrow, \downarrow\}$. With the three Pauli matrices $\sigma=\left(\sigma_{1}, \sigma_{2}, \sigma_{3}\right)$ and the identity $\sigma_{0}$ in spin space, we write $\hat{h}_{\boldsymbol{k}}=t_{0}\left(1-\cos k_{x}-\cos k_{y}\right) \sigma_{0}+\boldsymbol{b} \cdot \boldsymbol{\sigma}$, while the gap matrix components are $\Delta_{k, \uparrow \uparrow}=\Delta_{k, \downarrow \downarrow}^{*}=t_{0}\left(\sin k_{x}-i \sin k_{y}\right)$ and $\Delta_{k, \downarrow \uparrow}=-\Delta_{k, \uparrow \downarrow}=s_{x} \cos k_{x}+s_{y} \cos k_{y}$. The vectors $\boldsymbol{b} \equiv\left(b_{x}, b_{y}\right)$ and $\boldsymbol{s} \equiv\left(s_{x}, s_{y}\right)$ parametrize the in-plane magnetic field and the spin-singlet pairing amplitudes, respectively, with magnitudes smaller than $t_{0}$, taken as the unit in the following.

For $\boldsymbol{b}=\boldsymbol{s}=\mathbf{0}$, the Hamiltonian $\mathcal{H}$ describes electrons with nearest-neighbor hopping on a square lattice, subject to $p$ wave pairing. It possesses all three noncrystalline symmetries $(\mathcal{T}, \mathcal{P}$, and $\mathcal{C})$ and falls into the symmetry class DIII [9-11], supporting helical Majorana edge states [Fig. 2(a)]. Including crystalline symmetries, the total point symmetry group is $\mathcal{G}_{(\boldsymbol{b}=\mathbf{0}, \boldsymbol{s}=\mathbf{0})}=D_{4 h} \times\{\mathbb{1}, \mathcal{T}, \mathcal{P}, C\}$, where $\mathbb{1}$ represents the identity. This can be rewritten as $\mathcal{G}_{(\mathbf{0}, \mathbf{0})}=\tilde{\mathcal{G}}_{(\mathbf{0}, \mathbf{0})} \times\{\mathbb{1}, \mathcal{P}\}$, where the magnetic group reads $\tilde{\mathcal{G}}_{(\mathbf{0}, \mathbf{0})}=D_{4 h} \oplus \mathcal{T} D_{4 h}\left(\right.$ or $\left.4 / m m m 1^{\prime}\right)$ [for more details, see the Supplemental Material (SM) [70]].

A finite in-plane magnetic field $\boldsymbol{b}$ conserves $\mathcal{P}$ for any direction, but breaks $\mathcal{T}$. The reduced magnetic group is $\tilde{\mathcal{G}}_{(\boldsymbol{b}, \boldsymbol{0})}=C_{2 h} \oplus \mathcal{T}\left(D_{2 h}-C_{2 h}\right)$ [71] if $\boldsymbol{b}$ is parallel to $\hat{\boldsymbol{x}}, \hat{\boldsymbol{y}}$, or $\hat{\boldsymbol{x}} \pm \hat{\boldsymbol{y}}$, and $C_{i} \oplus \mathcal{T}\left(C_{2 h}-C_{i}\right)$ otherwise (i.e., $m^{\prime} m^{\prime} m$ and $2^{\prime} / m^{\prime}$, respectively). The magnetic field hybridizes the helical boundary modes unless they are protected by symmetry: As illustrated in Fig. 2(b), for $\boldsymbol{b} \| \hat{\boldsymbol{x}}$ only the $x$ edges remain metallic protected by $C \mathcal{M}_{y} \in \mathcal{G}_{(\hat{\boldsymbol{x}}, \mathbf{0})}$, a product of $C$ and the

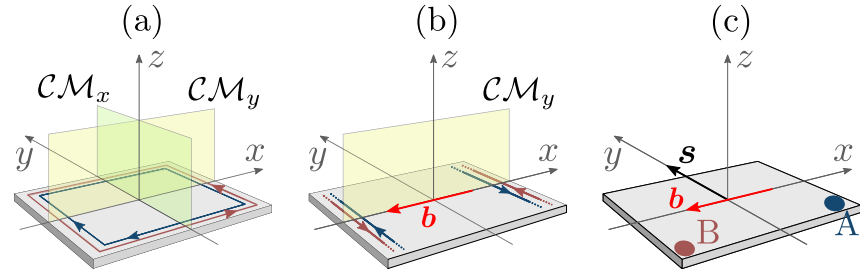

FIG. 2. (a) Schematic diagram for the helical state with two counterpropagating edge modes of opposite spins. At the $x(y)$ boundary, they are protected by the mirror antisymmetry $\mathcal{C M}_{y}$ $\left(C \mathcal{M}_{x}\right)$, a product of chiral symmetry and the mirror reflection $\mathcal{M}_{y}\left(\mathcal{M}_{x}\right)$. (b) The magnetic field $\boldsymbol{b}=(-0.3,0)$ breaks the mirror antisymmetry $\mathcal{C M}_{x}$ and gaps the $y$ edges. (c) If, additionally, the parameter $\boldsymbol{s}=\left(0, s_{y}\right) \| \hat{\boldsymbol{y}}$ is nonvanishing, the pairing term $\Delta_{\boldsymbol{k}, \downarrow \uparrow}=$ $s_{y} \cos k_{y}$ breaks the $C \mathcal{M}_{y}$ antisymmetry and two zero-energy modes appear, localized at two adjacent corners.

mirror reflection $\mathcal{M}_{y}$ which maps $(x, y, z) \mapsto(x,-y, z)$. Analogously, for $\boldsymbol{b} \| \hat{\boldsymbol{y}}$ the gapless states at the $y$ edges are protected by $\mathrm{CM}_{x}$ (see SM [70]). Both edges are gapped for generic orientations $\boldsymbol{b} \nVdash \hat{\boldsymbol{x}}, \hat{\boldsymbol{y}}$, but, if $\boldsymbol{b} \|(\hat{\boldsymbol{x}} \pm \hat{\boldsymbol{y}})$, the mirror-symmetric corners bisected by $\boldsymbol{b}$ will host zero-energy modes [28,72].

The spin-singlet pairing controlled by $\boldsymbol{s}(|\boldsymbol{s}| \neq 0)$ reduces the symmetry further and in the generic case $\tilde{\mathcal{G}}_{(b, s)}=C_{1} \oplus$ $\mathcal{T}\left(C_{s}-C_{1}\right)$ (or $\left.m^{\prime}\right)$. Thus, the (minimal) total group of the BdG Hamiltonian is $\mathcal{G}_{(b, s)}=\left\{\mathbb{1}, \mathcal{T} \mathcal{M}_{z}\right\} \times\{\mathbb{1}, \mathcal{P}\}$, including two particularly important elements, $\mathcal{P}$ and the effective chiral symmetry $\widetilde{C}=C \mathcal{M}_{z}=\mathcal{P} \mathcal{T} \mathcal{M}_{z}$, which transform the Hamiltonian in the following way,

$$
\begin{aligned}
& \mathcal{H}(\boldsymbol{k})=-U_{\mathcal{P}} \mathcal{H}(-\boldsymbol{k})^{*} U_{\mathcal{P}}^{\dagger}, \quad U_{\mathcal{P}}=\sigma_{3} \tau_{1}, \\
& \mathcal{H}(\boldsymbol{k})=-U_{\widetilde{C}} \mathcal{H}(+\boldsymbol{k}) U_{\widetilde{C}}^{\dagger}, \quad U_{\widetilde{\mathcal{C}}}=\sigma_{2} \tau_{1},
\end{aligned}
$$

where the Pauli matrix $\tau_{1}$ acts on the particle-hole space.

Majorana corner states. On a finite square-shaped lattice, the $\mathcal{P}$-symmetric Hamiltonian hosts two degenerate zero-energy excitations $\left|\psi_{\alpha}\right\rangle$, with $\alpha \in\{\mathrm{A}, \mathrm{B}\}$, whose features in the second-order topological phase are displayed in Fig. 1(a) for $\boldsymbol{b}=(-0.3,0)$ and $\boldsymbol{s}=(0,0.3)$. In this regime, although the magnetic field $\boldsymbol{b} \| \hat{\boldsymbol{x}}$ would allow for $\boldsymbol{C M}_{y^{-}}$ protected boundary modes at the $x$ edges, the spin-singlet pairing controlled by $\boldsymbol{s} \| \hat{\boldsymbol{y}}$ gaps the $x$ edges by breaking this symmetry [Fig. 2(c)] and enforces the zero-energy modes to localize at two adjacent corners of the device [as shown in the inset of Fig. 1(a)]. More precisely, the pairing term $\Delta_{k, \downarrow \uparrow}$ reduces $\tilde{G}_{(\hat{\boldsymbol{x}}, \mathbf{0})}=C_{2 h} \oplus \mathcal{T}\left(D_{2 h}-C_{2 h}\right)$ to $\tilde{G}_{(\hat{\boldsymbol{x}}, \hat{\boldsymbol{y}})}=C_{s} \oplus$ $\mathcal{T}\left(C_{2 v}-C_{s}\right)$ (i.e., $m^{\prime} m^{\prime} m$ to $\left.m^{\prime} m 2^{\prime}\right)$.

The operators corresponding to the MCSs can be written as

$$
\gamma_{\alpha}=\sum_{\boldsymbol{r}} \sum_{i=1}^{4}\left[\psi_{\alpha, r}\right]_{i}^{*}\left[\hat{\Psi}_{\boldsymbol{r}}\right]_{i}, \quad \alpha \in\{\mathrm{A}, \mathrm{B}\}
$$

where $\left[\hat{\Psi}_{\boldsymbol{r}}\right]_{i}$ is the $i$ th component of the Fourier-transformed Nambu basis for the site at position $\boldsymbol{r}$ and $\left[\psi_{\alpha, r}\right]_{i}$ the $i$ th component of the corresponding sector $\left|\psi_{\alpha, r}\right\rangle$ of the MCS $\left|\psi_{\alpha}\right\rangle$. Because $\left|\psi_{\alpha}\right\rangle$ are self-conjugate under $\mathcal{P}\left(\mathcal{P}\left|\psi_{\alpha, \boldsymbol{r}}\right\rangle \equiv\right.$ 


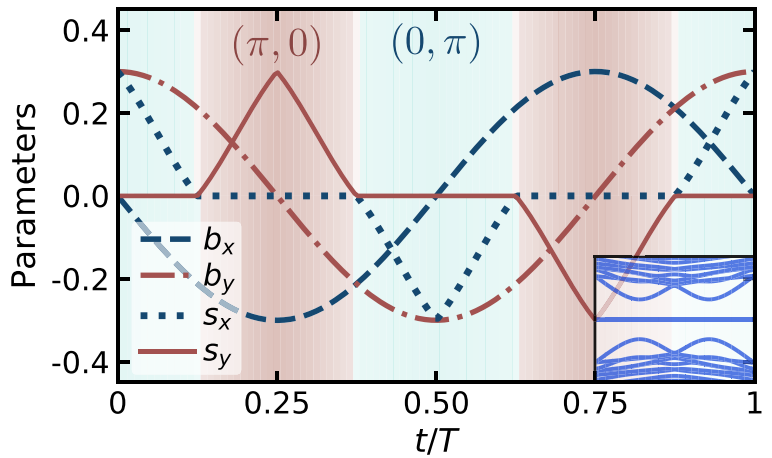

FIG. 3. The evolution of the Hamiltonian parameters $\boldsymbol{b} \equiv$ $\left(b_{x}, b_{y}\right)$ and $s \equiv\left(s_{x}, s_{y}\right)$ during the cyclic adiabatic process of period $T$. The alternating dark-light background colors correspond to second-order topological phases with invariants $\left(\phi_{x y}, \phi_{y x}\right)=(\pi, 0)$ or $(0, \pi)$, respectively. The inset displays the energy eigenvalues of the finite lattice in the range $E \in[-0.4,0.4]$, during the last quarter of the cyclic process (the period of the eigenvalues is $T / 4$ ).

$\left.U_{\mathcal{P}}\left|\psi_{\alpha, \boldsymbol{r}}\right\rangle^{*}=\left|\psi_{\alpha, \boldsymbol{r}}\right\rangle\right)$, these operators satisfy the following relations (for details, see SM [70]),

$$
\gamma_{\alpha}=\gamma_{\alpha}^{\dagger}, \quad\left\{\gamma_{\alpha}, \gamma_{\beta}\right\}=2 \delta_{\alpha \beta} \text {. }
$$

The energies of the midgap states go to zero exponentially with the system size (see SM [70]), are robust against $\mathcal{P}$-preserving disorder (tested numerically) and are well separated from the bulk states by an energy gap [emphasized in Fig. 1(a)]. The localization of the boundary modes is strongly dependent on the Hamiltonian parameters $\boldsymbol{b}$ and $\boldsymbol{s}$, whereby their energy remains zero for all the considered configurations, in which the device is in a SOTS phase. This degenerate ground-state manifold is crucial to realize the braiding of the topological MCSs.

Computing topological invariants. We can assess the topological features of our device using the Wilson loop operator (WLO), presented in more detail in the SM [70]. The WLO can be adiabatically connected to the Hamiltonian at the boundary [73-76] and can thus characterize the surface topology of materials, such as $\mathbb{Z}_{2}$ topological (crystalline or not) insulators in $2 \mathrm{D}$ and $3 \mathrm{D}[3,76-82]$ or $\mathbb{Z}$ Chern insulators [80,82-84].

Moreover, the nested WLO introduced in Ref. [25] has been used to address the physics at "the boundary of the boundary" [27,40,85]. In our 2D model, using the nested WLOs we obtain two $\mathbb{Z}_{2}$ corner topological invariants $\phi_{x y}, \phi_{y x} \in\{0, \pi\}$, which are quantized by the effective chiral symmetry introduced in Eq. (2b) (confirmed by numerical simulations). The device is in a SOTS phase whenever either of $\left\{\phi_{x y}, \phi_{y x}\right\}$ is equal to $\pi$; for example, in the case $\boldsymbol{b}=(-0.3,0)$ and $\boldsymbol{s}=(0,0.3)$, shown in Fig. 1(a), we have $\left(\phi_{x y}, \phi_{y x}\right)=(\pi, 0)$.

Braiding process. We perform the adiabatic braiding of the MCSs by rotating the magnetic field $(\boldsymbol{b})$ and adjusting the spin-singlet pairing $(s)$. Let $T$ be the time period of the cyclic process and $t \in[0, T]$ the parameter which controls the process, such that we have periodic boundary conditions $\mathcal{H}^{(t=0)}=\mathcal{H}^{(t=T)}$. The evolution of the parameters $\boldsymbol{b}(t)$ and $s(t)$ over a full period is shown in Fig. 3, where the alter-

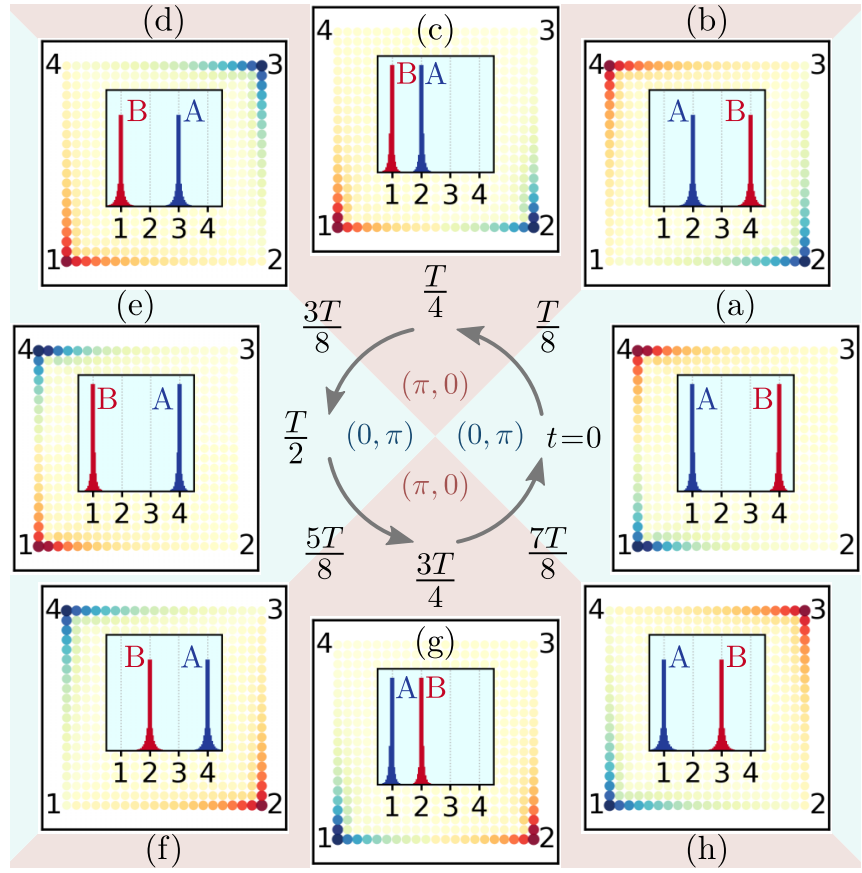

FIG. 4. Square lattices with $21 \times 21$ sites, displaying the spatial probability density $P_{\mathrm{A}}(t ; x, y)$ (blue color) and $P_{\mathrm{B}}(t ; x, y)$ (red color) of the two zero modes $\mathrm{A}$ and $\mathrm{B}$ during the adiabatic cycle, at time $t_{\mathrm{a}}=0$ in (a), $t_{\mathrm{b}}=T / 8$ in (b), $t_{\mathrm{c}}=2 T / 8$ in (c), etc. The probability of localization is maximum at the corners of the lattice (labeled with 1, 2, 3, and 4), which can be seen from the intensity of the colors. This feature is emphasized in the insets, where we used the parametrization $(x, y)=r(\cos \varphi, \sin \varphi)$ and plotted the angular probability distributions $P_{\alpha}(t ; \varphi)$ for $\alpha \in\{\mathrm{A}, \mathrm{B}\}$. The background colors are alternating when $t \in[0, T]$ and have the same meaning as in Fig. 3: The second-order topological invariants $\left(\phi_{x y}, \phi_{y x}\right)$ are equal to $(0, \pi)$ in the light regions and to $(\pi, 0)$ in the darker regions.

nations of the second-order topological states characterized by $\left(\phi_{x y}, \phi_{y x}\right)=(\pi, 0)$ and $(0, \pi)$ are also highlighted. Along this path in parameter space, the bulk and the edges remain gapped, while the energy of the degenerate MCSs is unaffected-they remain well separated from the bulk states by an energy gap (feature displayed in the inset of Fig. 3). The MCSs thus do not mix with the bulk states during the cyclic process, which we illustrate in Fig. 4 by computing the spatial probability density of $\left|\psi_{\alpha}(t)\right\rangle$ in the proposed device for several $t(\alpha \in\{\mathrm{A}, \mathrm{B}\})$.

Statistical phase. A fermionic many-body wave function picks a factor $(-1)$ upon exchanging two fermions and it reverts to the initial wave function if the exchange of any other two is performed. In contradistinction, in a system with $2 M$ Majorana quasiparticles $(M>1)$ two subsequent exchanges (say, $\gamma_{1} \leftrightarrow \gamma_{2}$ and $\gamma_{2} \leftrightarrow \gamma_{3}$ ) generally give a different state (non-Abelian braid group) [49-52]. In particular, if the system contains a single pair of Majoranas $(M=1)$, such a double exchange (braiding) reveals the fractional statistics of the two quasiparticles: Each acquires a nontrivial statistical phase $\Phi=\pi$.

The device we propose here realizes the braiding of two midgap states $\psi_{\alpha}$ [with corresponding operators $\gamma_{\alpha}, \alpha \in$ $\{$ A, B\}, see Eq. (3)] and we show below their statistics is indeed that of Majorana quasiparticles (fractional, $M=1$ ). We 
note, however, that in the proposed setup the single exchange $\mathrm{A} \leftrightarrow \mathrm{B}$ is not feasible with the parameter cycle considered in Fig. 3. Although at $t=T / 2$ the centers of the wave functions $\psi_{\mathrm{A}}, \psi_{\mathrm{B}}$ are swapped (see Fig. 4), the Hamiltonian after a half-period is different from the initial one. Consequently, a statistical phase between $\psi_{\mathrm{A}}\left(\psi_{\mathrm{B}}\right)$ at $t=0$ and $\psi_{\mathrm{B}}\left(\psi_{\mathrm{A}}\right)$ at $t=T / 2$ would not be well defined nor robust.

To express the braiding phase accumulated by each MCS within one full cycle (i.e., after a double exchange), we use the Berry phase defined as

$$
\Phi_{\alpha}=\oint_{0}^{T}\left[\mathcal{A}_{t}\right]_{\alpha \alpha} d t, \quad \alpha \in\{\mathrm{A}, \mathrm{B}\}
$$

where the Berry connection matrix has elements $\left[\mathcal{A}_{t}\right]_{\alpha \beta}=$ $i\left\langle\psi_{\alpha}(t)\left|\partial_{t}\right| \psi_{\beta}(t)\right\rangle$ (more details are given in the SM [70]). The quantity in Eq. (5) is gauge invariant because (i) the Hamiltonian parameter configurations at $t \in\{0, T\}$ are identical and (ii) the matrix $\mathcal{A}_{t}$ is diagonal $\forall t \in[0, T]$, as the two MCSs are kept far apart from each other and do not mix during the course of the adiabatic cycle. The practically null spatial overlap of the two states A and B was checked numerically and is illustrated in Figs. 1(b) and 4.

With Eq. (5), we obtain nontrivial statistical phases $\Phi_{\mathrm{A}}=$ $\Phi_{\mathrm{B}}=\pi$, which means

$$
\begin{aligned}
& \gamma_{\mathrm{A}} \rightarrow-\gamma_{\mathrm{A}}, \\
& \gamma_{\mathrm{B}} \rightarrow-\gamma_{\mathrm{B}},
\end{aligned}
$$

and, together with the operator algebra (4), proves the Majorana nature of the quasiparticles. As confirmed numerically, the quantization of the phases is guaranteed by $\mathcal{P}$, Eq. (2a).

However, the relative phase described here would be an inaccessible experimental quantity within the proposed protocol, since the two MCSs necessarily have different fermion parities. Nonetheless, we believe it might be possible to extend our scheme as to construct a system hosting $M>1$ Majorana pairs (for example, by distributing $M$ devices as a chain or a two-dimensional array). In such a setup, we would expect that the fractional statistics (6) of our MCSs $(M=1)$ would generalize to a non-Abelian braid group.

Schematic device design. Finally, we briefly describe a possible structure for the proposed device that would consist of three square-shaped thin-film superconductors stacked as in Fig. 5(a). The superconducting state in our model $\left(\hat{\Delta}_{k}\right)$ can be realized if we induce spin-singlet pairing in the $p$-wave film through the proximity effect, by placing it between an extended $s$-wave superconductor and a $d_{x^{2}-y^{2}}$-wave superconductor, with even-parity gap functions $\psi_{s / d}=\eta_{s / d}\left(\cos k_{x} \pm\right.$ $\cos k_{y}$ ). In terms of the $s$ parameter, the gap magnitudes can be written as $\eta_{s / d}=\left(s_{x} \pm s_{y}\right) / 2$. The relative phases between the superconductors can be fixed by Josephson contacts, as explained in the following.

First, the order parameter $\eta_{p}$ corresponding to the $p$ wave couples only in second order to the spin-singlet ones, which locks the $p-s$ and $p-d$ relative phases to either $\pi / 2$ or $-\pi / 2$ (in order to minimize the Josephson energy, in lowest order being typically $E_{\mathrm{J}} \propto+\cos 2 \phi_{p-s / d}$ ). Indeed, first-order coupling terms as $\left(\eta_{s / d}^{*} \eta_{p}+\right.$ c.c. $)$ are assumed to be absent or extremely weak, due to the mismatch of parity. Note that the $p$-wave superconducting state, $d$-vector $\boldsymbol{d}_{\boldsymbol{k}}=\hat{\boldsymbol{x}} \sin k_{y}-\hat{\boldsymbol{y}} \sin k_{x}$, belongs to irreducible representation $A_{2 u}$, while the order parameters

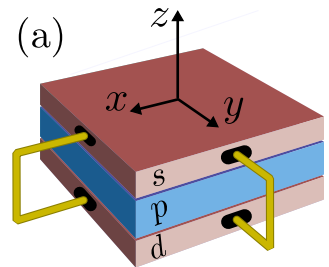

(b)
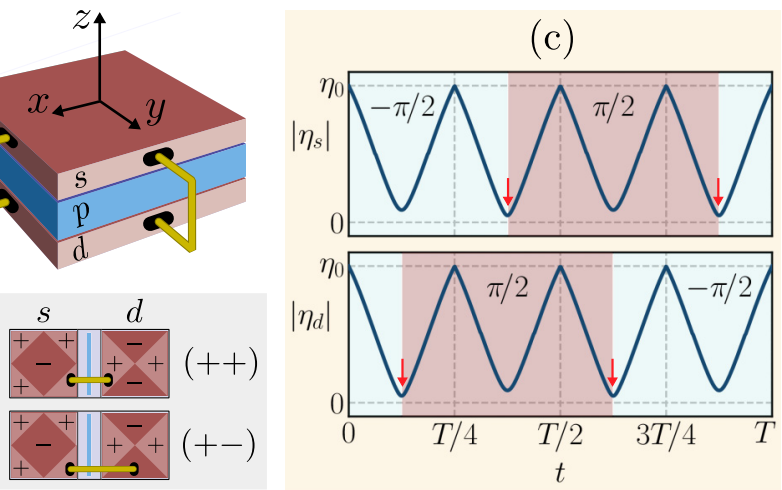

FIG. 5. (a) Schematic representation of the proposed device, consisting of a two-dimensional helical $p$-wave superconductor in contact with an extended $s$-wave and a $d$-wave superconductor. (b) Diagrams of the spin-singlet gap structures in the $x y$ plane, with positive and negative sectors. One of the Josephson contacts between the $s$ and $d$ superconductors [depicted as well in (a)] links two sectors of the same sign (++ configuration), while the other links sectors of different signs $(+-)$. (c) The magnitudes of the spin-singlet gap functions during the proposed adiabatic cycle of period $T$, with $+\pi / 2(-\pi / 2)$ phases relative to the $p$-wave superconductor in the $t$ intervals with dark-red (light-blue) background color. One commutes between the $(++)$ and $(+-)$ configurations when both $\left|\eta_{s / d}\right|$ are small; the red arrows indicate the smaller gap magnitude and thus the spin-singlet superconductor which flips the phase relative to the $p$ wave.

$\psi_{s}$ and $\psi_{d}$ belong to the even-parity representations $A_{1 g}$ and $B_{1 g}$, respectively. Thus, the phase coupling between the oddparity and even-parity order parameter would be dominated by the second-order coupling of the form $\left(\eta_{s / d}^{* 2} \eta_{p}^{2}+\right.$ c.c. $)$. The appropriate phase factor $e^{i \pi / 2}$ is already considered in Eq. (1), where the configuration " $\eta_{s}+\eta_{d}+i \eta_{p}$ " is presented.

Second, the relative phase between the spin-singlet condensates can be controlled using alternatively two direct Josephson contacts [see Fig. 5(a)]. The gap functions $\psi_{s}$ and $\psi_{d}$ exhibit in the $x y$ plane positive and negative sectors, as schematically represented in Fig. 5(b), and the Josephson contacts are set up such that they link equal- or different-sign sectors, respectively. Switching the active contact increases by $\pi$ not only the relative $s-d$ phase, but consequently also that singlet-triplet phase, where the Josephson coupling is weaker. As emphasized in Fig. 5(c), during the proposed adiabatic cycle such an operation changes the $p-s(p-d)$ phase by $\pi$ when $\left|\eta_{s}\right|<\left|\eta_{d}\right|\left(\left|\eta_{s}\right|>\left|\eta_{d}\right|\right)$.

Manipulating the spin-singlet gap magnitudes $\eta_{s / d}$ during the proposed cycle remains a challenging task. At a conceptual level, two strategies could be taken under consideration: using tunable tunneling Josephson contacts between the $p$ wave and the spin-singlet superconductors or modifying the order parameter amplitudes $\eta_{s}$ and $\eta_{d}$ individually by local heating or other means. In fact, small changes to the proposed functions $\eta_{s / d}(t)$, that might come within the experimental implementation, are not expected to change the topological features of the cycle. The latter argument applies as well to the in-plane magnetic field $\boldsymbol{b}(t)$. Moreover, we assume this to be weak enough such that in the superconducting thin films the 
interference with the Josephson effect as well as the depairing effects are negligible.

Acknowledgments. T.E.P. would like to thank J. L. Lado, T. Kawakami, and K. Viebahn for helpful discussions. T.E.P. and M.S. are grateful for the financial support from the
Swiss National Science Foundation (SNSF) through Division II (No. 163186 and No. 184739). A.A.S. acknowledges the support of Microsoft Research, SNSF NCCR MARVEL and QSIT programs, and the SNSF Professorship grant.
[1] C. L. Kane and E. J. Mele, Phys. Rev. Lett. 95, 146802 (2005).

[2] B. A. Bernevig, T. L. Hughes, and S.-C. Zhang, Science 314, 1757 (2006)

[3] L. Fu and C. L. Kane, Phys. Rev. B 74, 195312 (2006).

[4] L. Fu, C. L. Kane, and E. J. Mele, Phys. Rev. Lett. 98, 106803 (2007).

[5] L. Fu and C. L. Kane, Phys. Rev. B 76, 045302 (2007).

[6] M. Z. Hasan and C. L. Kane, Rev. Mod. Phys. 82, 3045 (2010).

[7] X.-L. Qi and S.-C. Zhang, Rev. Mod. Phys. 83, 1057 (2011).

[8] C.-K. Chiu, J. C. Y. Teo, A. P. Schnyder, and S. Ryu, Rev. Mod. Phys. 88, 035005 (2016).

[9] A. P. Schnyder, S. Ryu, A. Furusaki, and A. W. W. Ludwig, Phys. Rev. B 78, 195125 (2008).

[10] A. P. Schnyder, S. Ryu, A. Furusaki, and A. W. W. Ludwig, in Advances in Theoretical Physics: Landau Memorial Conference, edited by V. Lebedev and M. Feigel'man, AIP Conf. Proc. No. 1134 (AIP, Melville, NY, 2009), p. 10.

[11] A. Kitaev, in Advances in Theoretical Physics: Landau Memorial Conference (Ref. [10]), p. 22.

[12] L. Fu, Phys. Rev. Lett. 106, 106802 (2011).

[13] T. H. Hsieh, H. Lin, J. Liu, W. Duan, A. Bansil, and L. Fu, Nat. Commun. 3, 982 (2012).

[14] R.-J. Slager, A. Mesaros, V. Juričić, and J. Zaanen, Nat. Phys. 9, 98 (2012).

[15] C.-K. Chiu, H. Yao, and S. Ryu, Phys. Rev. B 88, 075142 (2013).

[16] T. Morimoto and A. Furusaki, Phys. Rev. B 88, 125129 (2013).

[17] K. Shiozaki and M. Sato, Phys. Rev. B 90, 165114 (2014).

[18] W. A. Benalcazar, J. C. Y. Teo, and T. L. Hughes, Phys. Rev. B 89, 224503 (2014).

[19] A. Alexandradinata, C. Fang, M. J. Gilbert, and B. A. Bernevig, Phys. Rev. Lett. 113, 116403 (2014).

[20] K. Shiozaki, M. Sato, and K. Gomi, Phys. Rev. B 93, 195413 (2016).

[21] J. Kruthoff, J. de Boer, J. van Wezel, C. L. Kane, and R.-J. Slager, Phys. Rev. X 7, 041069 (2017).

[22] K. Shiozaki, M. Sato, and K. Gomi, Phys. Rev. B 95, 235425 (2017).

[23] R. Thorngren and D. V. Else, Phys. Rev. X 8, 011040 (2018).

[24] E. Khalaf, H. C. Po, A. Vishwanath, and H. Watanabe, Phys. Rev. X 8, 031070 (2018).

[25] W. A. Benalcazar, B. A. Bernevig, and T. L. Hughes, Science 357, 61 (2017).

[26] Z. Song, Z. Fang, and C. Fang, Phys. Rev. Lett. 119, 246402 (2017).

[27] F. Schindler, A. M. Cook, M. G. Vergniory, Z. Wang, S. S. P. Parkin, B. A. Bernevig, and T. Neupert, Sci. Adv. 4, eaat0346 (2018).

[28] M. Geier, L. Trifunovic, M. Hoskam, and P. W. Brouwer, Phys. Rev. B 97, 205135 (2018).

[29] E. Khalaf, Phys. Rev. B 97, 205136 (2018).

[30] J. Ahn and B.-J. Yang, Phys. Rev. B 99, 235125 (2019).
[31] G. van Miert and C. Ortix, Phys. Rev. B 98, 081110(R) (2018).

[32] W. A. Benalcazar, B. A. Bernevig, and T. L. Hughes, Phys. Rev. B 96, 245115 (2017).

[33] J. Langbehn, Y. Peng, L. Trifunovic, F. von Oppen, and P. W. Brouwer, Phys. Rev. Lett. 119, 246401 (2017).

[34] Y. Wang, M. Lin, and T. L. Hughes, Phys. Rev. B 98, 165144 (2018).

[35] D. Călugăru, V. Juričić, and B. Roy, Phys. Rev. B 99, 041301(R) (2019).

[36] S. A. A. Ghorashi, X. Hu, T. L. Hughes, and E. Rossi, Phys. Rev. B 100, 020509(R) (2019).

[37] A. Agarwala, V. Juričić, and B. Roy, Phys. Rev. Research 2 , 012067(R) (2020).

[38] F. Schindler, Z. Wang, M. G. Vergniory, A. M. Cook, A. Murani, S. Sengupta, A. Y. Kasumov, R. Deblock, S. Jeon, I. Drozdov, H. Bouchiat, S. Guéron, A. Yazdani, B. A. Bernevig, and T. Neupert, Nat. Phys. 14, 918 (2018).

[39] Y. Volpez, D. Loss, and J. Klinovaja, Phys. Rev. Lett. 122, 126402 (2019).

[40] S. Franca, J. van den Brink, and I. C. Fulga, Phys. Rev. B 98 201114(R) (2018).

[41] M. Rodriguez-Vega, A. Kumar, and B. Seradjeh, Phys. Rev. B 100, 085138 (2019).

[42] B. Huang and W. V. Liu, Phys. Rev. Lett. 124, 216601 (2020).

[43] M. Serra-Garcia, V. Peri, R. Süsstrunk, O. R. Bilal, T. Larsen, L. G. Villanueva, and S. D. Huber, Nature (London) 555, 342 (2018).

[44] C. W. Peterson, W. A. Benalcazar, T. L. Hughes, and G. Bahl, Nature (London) 555, 346 (2018).

[45] S. Imhof, C. Berger, F. Bayer, J. Brehm, L. W. Molenkamp, T. Kiessling, F. Schindler, C. H. Lee, M. Greiter, T. Neupert, and R. Thomale, Nat. Phys. 14, 925 (2018).

[46] M. Ezawa, Phys. Rev. Lett. 120, 026801 (2018).

[47] C.-H. Hsu, P. Stano, J. Klinovaja, and D. Loss, Phys. Rev. Lett. 121, 196801 (2018).

[48] A. Y. Kitaev, Phys. Usp. 44, 131 (2001).

[49] A. Kitaev, Ann. Phys. 321, 2 (2006).

[50] C. Nayak, S. H. Simon, A. Stern, M. Freedman, and S. DasSarma, Rev. Mod. Phys. 80, 1083 (2008).

[51] J. Alicea, Rep. Prog. Phys. 75, 076501 (2012).

[52] S. D. Sarma, M. Freedman, and C. Nayak, npj Quantum Inf. 1, 15001 (2015)

[53] L. Fidkowski and A. Kitaev, Phys. Rev. B 81, 134509 (2010).

[54] L. Fidkowski and A. Kitaev, Phys. Rev. B 83, 075103 (2011).

[55] R. M. Lutchyn, J. D. Sau, and S. DasSarma, Phys. Rev. Lett. 105, 077001 (2010).

[56] Y. Oreg, G. Refael, and F. von Oppen, Phys. Rev. Lett. 105, 177002 (2010).

[57] V. Mourik, K. Zuo, S. M. Frolov, S. R. Plissard, E. P. A. M. Bakkers, and L. P. Kouwenhoven, Science 336, 1003 (2012).

[58] A. Das, Y. Ronen, Y. Most, Y. Oreg, M. Heiblum, and H. Shtrikman, Nat. Phys. 8, 887 (2012). 
[59] M. T. Deng, C. L. Yu, G. Y. Huang, M. Larsson, P. Caroff, and H. Q. Xu, Nano Lett. 12, 6414 (2012).

[60] A. D. K. Finck, D. J. VanHarlingen, P. K. Mohseni, K. Jung, and X. Li, Phys. Rev. Lett. 110, 126406 (2013).

[61] H. O. H. Churchill, V. Fatemi, K. Grove-Rasmussen, M. T. Deng, P. Caroff, H. Q. Xu, and C. M. Marcus, Phys. Rev. B 87, 241401(R) (2013).

[62] M. T. Deng, S. Vaitiekènas, E. B. Hansen, J. Danon, M. Leijnse, K. Flensberg, J. Nygård, P. Krogstrup, and C. M. Marcus, Science 354, 1557 (2016).

[63] J. Alicea, Y. Oreg, G. Refael, F. von Oppen, and M. P. A. Fisher, Nat. Phys. 7, 412 (2011).

[64] B. van Heck, A. R. Akhmerov, F. Hassler, M. Burrello, and C. W. J. Beenakker, New J. Phys. 14, 035019 (2012).

[65] X. Zhu, Phys. Rev. B 97, 205134 (2018).

[66] Z. Yan, F. Song, and Z. Wang, Phys. Rev. Lett. 121, 096803 (2018).

[67] Q. Wang, C.-C. Liu, Y.-M. Lu, and F. Zhang, Phys. Rev. Lett. 121, 186801 (2018)

[68] T. Liu, J. J. He, and F. Nori, Phys. Rev. B 98, 245413 (2018).

[69] C. Schrade and L. Fu, arXiv:1807.06620.

[70] See Supplemental Material at http://link.aps.org/supplemental/ 10.1103/PhysRevResearch.2.032068 for more details regarding the system symmetries and the computation of topological invariants.

[71] Here, the set $\left(D_{2 h}-C_{2 h}\right)$ represents the relative complement of $C_{2 h}$ with respect to $D_{2 h}$. It contains the elements of $D_{2 h}$ which are not part of $C_{2 h}$ and it is not a group by itself.
[72] M. Ezawa, Phys. Rev. Lett. 121, 116801 (2018).

[73] E. I. Blount, in Solid State Physics, Vol. 13, edited by F. Seitz and D. Turnbull (Academic Press, New York, London, 1962), pp. 305-373.

[74] R. D. King-Smith and D. Vanderbilt, Phys. Rev. B 47, 1651 (1993).

[75] L. Fidkowski, T. S. Jackson, and I. Klich, Phys. Rev. Lett. 107, 036601 (2011).

[76] M. Taherinejad, K. F. Garrity, and D. Vanderbilt, Phys. Rev. B 89, 115102 (2014).

[77] A. A. Soluyanov and D. Vanderbilt, Phys. Rev. B 83, 035108 (2011).

[78] A. A. Soluyanov and D. Vanderbilt, Phys. Rev. B 83, 235401 (2011).

[79] R. Yu, X. L. Qi, A. Bernevig, Z. Fang, and X. Dai, Phys. Rev. B 84, 075119 (2011).

[80] A. Alexandradinata, X. Dai, and B. A. Bernevig, Phys. Rev. B 89, 155114 (2014).

[81] A. Alexandradinata and B. A. Bernevig, Phys. Rev. B 93, 205104 (2016).

[82] D. Gresch, G. Autès, O. V. Yazyev, M. Troyer, D. Vanderbilt, B. A. Bernevig, and A. A. Soluyanov, Phys. Rev. B 95, 075146 (2017).

[83] S. Coh and D. Vanderbilt, Phys. Rev. Lett. 102, 107603 (2009).

[84] X.-L. Qi, Phys. Rev. Lett. 107, 126803 (2011).

[85] B.-Y. Xie, H.-F. Wang, H.-X. Wang, X.-Y. Zhu, J.-H. Jiang, M.-H. Lu, and Y.-F. Chen, Phys. Rev. B 98, 205147 (2018). 